\title{
PERITONITE ESCLEROSANTE ENCAPSULANTE EM RECEPTORA DE TRANSPLANTE RENAL - RELATO DE CASO
}

\author{
Encapsulating peritoneal sclerosis in recipient of kidney transplantation - \\ Case report
}

\author{
Vanessa Suemi Takenaka 1,2, Felipe Sbrolini Borges 1,2, Thales Franco de Andrade 1,2, André Petraroia Capelozza ${ }^{1,2}$, \\ Sibele Braga 1,3, Gabriel Castilho Schnorr 1,2, Laís Pacca Nicolellis 1,2, Jorge Marcelo Padilla Mancero 1,2,4, \\ Irene L. Noronha ${ }^{1,3,5}$, André Ibrahim David ${ }^{1,2,4}$
}

\section{RESUMO}

A peritonite esclerosante encapsulante (PEE) é uma condição rara e grave, frequentemente associada à diálise peritoneal. Apresenta-se como uma massa de tecido fibroso, recobrindo os tecidos viscerais, podendo comprometer o funcionamento fisiológico de todo o aparelho digestivo. O presente estudo relata um caso de PEE, em decorrência da diálise peritoneal (DP), devido à insuficiência renal por glomeruloesclerose segmentar e focal. No caso relatado, a paciente foi submetida à DP e passou por dois transplantes renais intervivos. São descritas as técnicas cirúrgicas que se revelaram úteis na resolução da obstrução intestinal, com melhora dos efeitos secundários da PEE. A dieta parenteral mostrou ser importante fator para a manutenção do aporte nutricional, auxiliando na cicatrização e no nível sérico de proteínas, vitaminas e eletrólitos. A terapia com tamoxifeno e a administração de hipossulfito de sódio foram eficientes para retardar o avanço da PEE.

Descritores: Peritonite; Diálise Peritoneal; Obstrução intestinal; Transplante de Órgãos; Transplante renal; Fibrose Peritoneal.

\section{Institution}

1. Hospital Beneficência Portuguesa de São Paulo - SP

2. Instituto de Gastrocirurgia Avançada do Hospital Beneficência Portuguesa de São Paulo-SP

3. Equipe de Nefrologia e Transplante do Hospital Beneficência Portuguesa de São Paulo-SP

4. Programa de Transplante de Fígado do Instituto de Gastrocirurgia Avançada do Hospital Beneficência Portuguesa de São Paulo-SP

5. Laboratório de Nefrologia Celular, Genética e Molecular da

Faculdade de Medicina da Universidade de São Paulo-SP

\section{Correspondência:}

Vanessa Suemi Takenaka

Rua Maestro Cardim, 377 - Cj.22 - CEP: 01323-000 - São Paulo/SP

Tel.: (11) 5575-7328

E-mail: suemi.takenaka@hotmail.com

\section{INTRODUÇÃO}

A peritonite esclerosante encapsulante (PEE) é uma complicação grave em pacientes submetidos à diálise peritoneal (DP), e rara, com prevalência de 0,7 a $3,7 \% .^{1}$ A ocorrência da PEE parece estar relacionada ao tempo de exposição do peritônio ao líquido dialítico, podendo chegar a $19,4 \%$ naqueles com mais de oito anos de tratamento. ${ }^{2}$ A mortalidade varia de $60-93 \% .^{3}$

PEE é caracterizada por um processo inflamatório crônico de etiologia desconhecida, resultado de peritonites bacterianas espontâneas recorrentes, que se manifestam de forma clínica ou subclínica. $O$ peritônio é gradualmente transformado em tecido fibroso difuso, resultando na formação de "folhas" ou "casulos" de tecido que recobrem e encapsulam as 
Vanessa Suemi Takenaka, Felipe Sbrolini Borges, Thales Franco de Andrade, André Petraroia Capelozza, Sibele Braga, Gabriel Castilho Schnorr, Laís Pacca Nicolellis, Jorge Marcelo Padilla Mancero, Irene L. Noronha, André Ibrahim David

vísceras, resultando em constrição visceral fibrótica. Como consequência, compromete a motilidade e altera o funcionamento do intestino, levando a graves complicações, como necrose intestinal, obstrução, fístula enterocutânea, sepse e óbito. ${ }^{1}$

Os principais fatores potenciais de risco para o desenvolvimento de PEE são a duração da DP e episódios repetidos de peritonites bacterianas espontâneas. ${ }^{4-6}$ No entanto, a PEE pode estar associada à exposição a asbestos, medicamentos (beta-bloqueadores, derivados do ergot, inibidores da calcineurina). ${ }^{7} \mathrm{Na}$ última década, houve aumento da incidência de PEE nos primeiros anos após transplante renal. ${ }^{8,9}$

Muitos casos de PEE não são relatados, principalmente em suas formas brandas. ${ }^{10}$ Geralmente, os sintomas apresentam-se como uma urgência com quadro de obstrução intestinal. No entanto, no início, há sintomas inespecíficos como dor abdominal, perda de peso, náuseas e vômitos. O diagnóstico de PEE requer dois critérios a ser preenchidos: características clínicas da obstrução e a demonstração, por imagem ou durante a cirurgia, de que as características clínicas são devidas ao espessamento da membrana peritoneal, resultando em encapsulamento do intestino. A realização da análise do anatomopatológico é feita em caso de tratamento cirúrgico ou remoção do cateter da diálise. Estudos radiológicos podem confirmar a $\mathrm{PEE}$, entretanto, não precoce o suficiente para sua prevenção. $O$ diagnóstico precoce, antes de ocorrer fibrose irreversível e encapsulamento, é extremamente importante para prevenção das complicações intestinais (obstrutivas). Os sintomas da PEE podem progredir após a interrupção da DP, transferência para hemodiálise ou transplante renal. ${ }^{11}$

Há ainda poucos relatos sobre a conduta cirúrgica nessa patologia; a maioria foi descrita no Japão (enterectomias, lise de aderências intestinais ou peritonectomia). ${ }^{12-16} \mathrm{~A}$ causa e patogênese exata da PEE ainda não são claras. A presença de duas etapas marcantes foi observada no desenvolvimento da PEE, como a exposição em longo prazo de soluções biocompatíveis com potencial de indução de fibrose e esclerose (primeira etapa), seguida de inflamação crônica (segunda etapa). ${ }^{5,6}$

\section{OBJETIVO}

O objetivo é relatar um caso de PEE, que surgiu em decorrência da DP e evoluiu com quadro de obstrução intestinal, em um paciente submetido previamente a dois transplantes renais. O presente relato analisa os benefícios das intervenções cirúrgicas para melhora da qualidade de vida e sintomatologia dessa patologia. A força deste relato é o ensinamento técnico e o alerta sobre essa delicada fase da adolescência, onde a não aderência ao tratamento é fato costumeiro.

\section{MATERIAL E MÉTODO}

Realizada revisão do prontuário, anamnese detalhada, registro fotográfico dos métodos diagnósticos $e$ terapêuticos ao qual a paciente foi submetida e revisão da literatura.

A revisão da literatura foi feita nas bases de dados do Medline, Bireme, Scielo, onde foram encontrados 59 trabalhos com os seguintes descritores: Encapsulating sclerosing peritonitis, Peritoneal dialysis complications, Intestinal obstruction, Renal transplant complications, Peritoneal Fibrosis; desses, 35 artigos foram selecionados.

\section{RELATO DE CASO}

Paciente do sexo feminino, 25 anos, nascida em março de 1989. Aos dois anos de idade, foi diagnosticada com glomeruloesclerose segmentar e focal e foi realizado tratamento com corticoide. Em 1992, aos três anos de idade, apresentou quadro de tetania, iniciando então DP, por sete meses.

Em fevereiro de 1993, aos quatro anos de idade, foi submetida ao primeiro transplante renal intervivos pela técnica convencional extraperitoneal, no qual sua mãe foi doadora. Evoluiu bem por 12 anos, até que no ano de 2005, com 16 anos de idade, passou a tomar os imunossupressores de maneira inadequada, ou seja, com má aderência ao tratamento pós-transplante renal. Evoluiu com rejeição tardia do enxerto, retornando para DP.

Em 2009, aos 20 anos de idade, foi submetida ao segundo transplante renal intervivos, também pela técnica convencional extraperitoneal, sendo o pai o doador. Evoluiu com rejeição hiperaguda e teve de ser submetida a quatro abordagens cirúrgicas durante essa internação, sendo a primeira para o implante do enxerto renal, a segunda para a realização de biópsia do enxerto, a terceira para a retirada do órgão e a quarta para a drenagem de cavidade por coleção. Ainda com 20 anos, reiniciou a DP. Durante o período total de DP, a paciente apresentou mais de cinco episódios de peritonites bacterianas espontâneas.

Em março de 2013, aos 24 anos de idade, evoluiu com náuseas, vômitos, diarréia, perda de peso e dor abdominal difusa, tendo sido internada. Nessa ocasião, apresentou saída de material com aspecto de areia, no efluente da DP. Em setembro de 2013, foi passada 
sonda nasogástrica e prescrita dieta parenteral. Em outubro de 2013, foi realizada biópsia de peritônio por vídeolaparoscopia, porém não foi confirmado o diagnóstico de peritonite esclerosante. A equipe que realizou a intervenção relatou que o aspecto era altamente sugestivo de esclerose de peritônio. Com a forte suspeita diagnóstica de PEE, em novembro de 2013 foi iniciado tratamento com prednisona $40 \mathrm{mg} / \mathrm{dia}$ e tamoxifeno $40 \mathrm{mg} / \mathrm{dia}$. Devido à ineficiência da DP, iniciou hemodiálise.

Em março de 2014, aos 25 anos de idade, foi internada no Hospital Beneficência Portuguesa de São Paulo, apresentando quadro de constipação intestinal, mantendo-se a suspeita de esclerose peritoneal devido à saída de material com aspecto de areia, no efluente da DP e características da cavidade em laparoscopia anterior, mesmo sem confirmação por biópsia. Foi iniciado tratamento com tiossulfato (hipossulfito de sódio $25 \%$ - $80 \mathrm{ml}$ diluídos em $200 \mathrm{ml}$ de SF 0,9\%), endovenoso, três vezes por semana, na última hora da sessão de hemodiálise. Apresentou boa resposta, com retorno da evacuação, tendo alta hospitalar assintomática. Após a parada do uso de hipossulfito de sódio, devido à dificuldade de acesso ao medicamento, a paciente voltou a apresentar o mesmo quadro intestinal.

Em agosto de 2014, foi internada novamente no serviço, com quadro de obstrução intestinal e piora progressiva. Foi realizada tomografia computadorizada (TC) do abdome total e pelve, em que pode ser observado espessamento difuso do peritônio parietal e visceral, com extensas calcificações, associada à ascite, calcificações envolvendo o mesentério e alças entéricas, sugestivo de peritonite esclerosante, além de moderado pneumoperitôneo e sinais de nefropatia crônica bilateral (Figura 1).
Figura 1

Corte sagital de TC de abdome e pelve demonstrando depósito de material em fundo de saco, espessamento e calcificação do peritônio parietal e visceral, calcificações lineares grosseiras, dilatação de alças intestinais, pneumoperitônio e ascite.

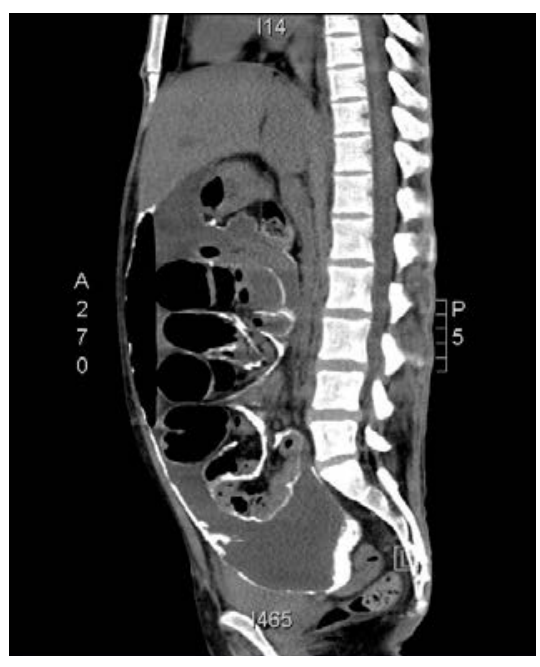

Foi introduzida dieta parenteral para melhora do aporte nutricional e realizada a videolaroscopia diagnóstica, que evidenciou grande quantidade de líquido purulento e estenose em íleo terminal e ceco. A cirurgia foi convertida e observou-se cavidade com calcificação grosseira e alteração da anatomia dos órgãos (Figura 2), principalmente do intestino delgado e grosso (Figura 3), confirmando obstrução intestinal.

Figura 2

Calcificações difusas no peritônio visceral das alças de intestino delgado

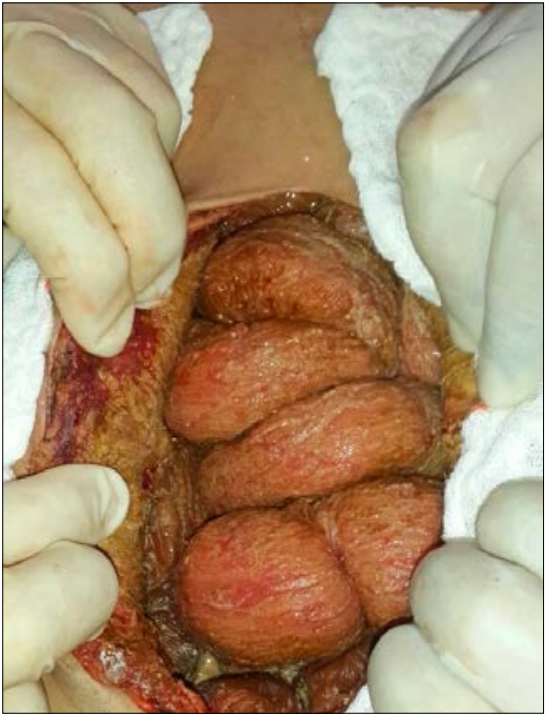

Figura 3 - Estenose total da luz intestinal do íleo terminal

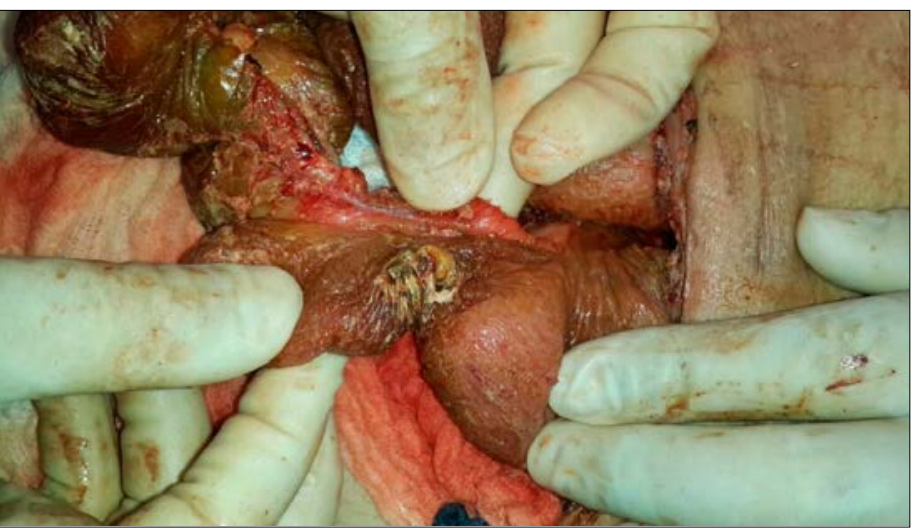

Procedeu-se à enterectomia segmentar do íleo terminal estenótico (Figura 4), com fechamento do cólon ascendente, ileostomia terminal, lavagem e drenagem da cavidade abdominal.

Figura 4

Segmento ressecado de íleo e cólon direito, com fibrose e estenose de íleostomia terminal.

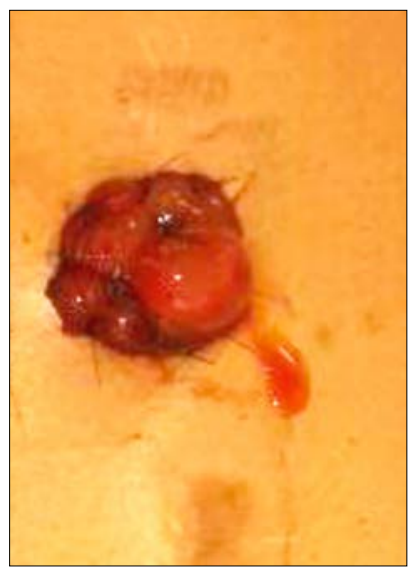


Vanessa Suemi Takenaka, Felipe Sbrolini Borges, Thales Franco de Andrade, André Petraroia Capelozza, Sibele Braga, Gabriel Castilho Schnorr, Laís Pacca Nicolellis, Jorge Marcelo Padilla Mancero, Irene L. Noronha, André Ibrahim David

O anatomopatológico da peça demonstrou serosite crônica fibrino-leucocitária em organização com fibrose e hialinização, associada a múltiplas áreas de calcificações distróficas e válvula íleo-cecal exibindo hipertrofia lipomatosa.

Após a primeira abordagem cirúrgica, evoluiu com a resolução do quadro de obstrução intestinal. Houve melhora do quadro infeccioso após a lavagem e drenagem da cavidade abdominal. A ileostomia mantevese funcionante, sendo realimentada por via oral.

No dia 8 de novembro de 2014, evoluiu com acidente vascular cerebral hemorrágico e no dia 9 de novembro de 2014, foi confirmada morte encefálica.

\section{DISCUSSÃO}

A paciente deste relato de caso foi submetida a dois transplantes renais intervivos. Após a falência dos dois enxertos, iniciou tratamento com DP desenvolvendo PEE.

Apresentava alguns fatores de risco associados, como longo período de DP (aproximadamente oito anos no total), episódios de peritonite bacteriana espontânea, uso inadequado de imunossupressores e dois transplantes renais.

Algumas limitações do presente relato devem ser levadas em consideração: a falta de informações precisas em prontuário referente ao total exato de episódios prévios de peritonite e imunossupressores utilizados e suas doses.

A patogênese dessa doença não está bem definida. Algumas das teorias são: o peritônio é pré-condicionado pelas soluções utilizadas na $\mathrm{DP}$, gerando uma inflamação aumentada e, após longo período de DP e peritonites bacterianas ou fúngicas, pode resultar em um processo de fibrose descontrolado e posterior encapsulamento dos intestinos. Nos pacientes submetidos a transplante renal, o trauma do transplante associado à inflamação causada pelas substâncias utilizadas na DP, podem resultar também na fibrose aumentada e no encapsulamento do intestino. Outra hipótese é que a PEE após o transplante pode estar relacionado ao uso concomitante de inibidores de calcineurina, substâncias pró-fibróticas. Além do fato de que, no peritônio inflamado, já existe uma regulação positiva do TGF- $\beta$ que leva a fibrose e neoangiogênese. ${ }^{17} \mathrm{E}$, tanto o tacrolimus como a ciclosporina também levam à maior expressão de TGF- $\beta$ e subsequente fibrose. ${ }^{18}$

As características clínicas dos pacientes com PEE na apresentação inicial podem ser não específicas, sendo a dor abdominal, perda de peso, náuseas e vômitos os sintomas iniciais mais comuns. ${ }^{19,20}$
O diagnóstico precoce, através da suspeita clínica em pacientes com obstrução intestinal e fatores de risco para $\mathrm{PEE}$, associado a algum método de imagem (radiológico ou cirúrgico), é fundamental para iniciar o mais rapidamente possível o tratamento e retardar o avanço da doença. ${ }^{19,20}$ Realizar lavagem cirúrgica da cavidade abdominal retirando as calcificações intraabdominais, prevenindo as infecções e protelando a obstrução intestinal.

Para o tratamento da PEE não há consenso definido. A imunossupressão com prednisolona, azatioprina ou micofenolato mofetil pós-transplante ou como terapia específica pode apresentar bons resultados. ${ }^{21,22}$

Diversos relatos na literatura indicam melhora da fibrose nos pacientes com PEE tratados com tamoxifeno. $O$ tamoxifeno, um modulador seletivo do receptor de estrógenos, pode representar nova opção terapêutica para promover o bloqueio de processos de fibrose. Há vários relatos que descrevem a eficácia do tamoxifeno na promoção da regressão da fibrose retroperitoneal idiopática e no tratamento da PEE. ${ }^{23-30}$

Os efeitos anti- fibróticos do tamoxifeno também foram descritos em outros modelos de doenças associadas à fibrose.30 Além das evidências clínicas de regressão da fibrose com o tratamento com tamoxifeno, estudos in vitro também sugeriram que o tamoxifeno possui propriedades antifibróticas. O tamoxifeno suprime a transcrição e síntese de colágeno, diminui a expressão de TGF-ß e inibe a proliferação de fibroblastos. ${ }^{17,18}$

O presente caso teve como agravante um processo de calcificação acentuada, inclusive da membrana peritoneal, sugerindo estado de calcifilaxia. Podem ser considerados fatores de risco para a calcifilaxia o metabolismo do cálcio e fósforo desregulados na doença renal crônica, o período prolongado em DP e o uso de quelantes de fósforo à base de cálcio em altas doses em longo prazo, alterações minerais ou hormonais. ${ }^{31-35}$

Para o tratamento de calcifilaxia, tem sido relatado o uso de tiossulfato de sódio endovenoso, ou mesmo intraperitoneal. O tiossulfato de sódio é um potente antioxidante e também aumenta a solubilidade dos depósitos de cálcio. As doses intravenosas variaram de 5-75 g após ou durante a hemodiálise em adultos. A dose mais comumente relatada foi de $25 \mathrm{~g}$ após cada diálise. Os tempos de infusão variam de 30 a 60 minutos. Embora geralmente bem tolerados, os efeitos adversos incluem náuseas e vômitos e acidose metabólica. ${ }^{34-36}$

Alguns estudos têm avaliado a DP com hipossulfito de sódio, a nutrição parenteral total, imunossupressão e o uso de Tamoxifeno com resultados positivos na 
maioria deles, o que foi também demostrado em nosso caso. A nutrição parenteral teve grande importância na manutenção do aporte nutricional, além de auxiliar na cicatrização, no nível sérico de proteínas, vitaminas e eletrólitos.

A intervenção cirúrgica demonstrou ser de grande valia no tratamento das complicações da PEE, além da lavagem da cavidade abdominal, que diminui a quantidade de calcificações intraperitoneais, levando à diminuição do foco infeccioso, como também dos quadros de obstrução intestinal, fístulas enterocutâneas, necrose intestinal, fibrose e calcificações vasculares intra-abdominais, que também são reduzidas significativamente após a intervenção cirúrgica, gerando melhora do quadro clínico e da qualidade de vida dos indivíduos portadores dessa patologia, permitindo realimentação oral e diminuindo a sintomatologia.

\section{CONCLUSÃO}

Em relação à $\mathrm{PEE}$, concluímos que é de extrema importância o tratamento cirúrgico, tanto para retirar o paciente do quadro de obstrução intestinal, uma complicação conhecida dessa patologia, quanto para lavar a cavidade abdominal e retirar as calcificações, fato que permitiu a realimentação da paciente e comprovou a melhora da qualidade de vida. O tratamento coadjuvante com tamoxifeno e tiosulfato podem representar alternativas terapêuticas para controlar o processo de fibrogênese e calcifilaxia.

\section{ABSTRACT}

Encapsulating Peritoneal Sclerosis (EPS) is a rare and severe condition often associated to Peritoneal Dialysis (PD). It is presented as a mass of fibrous tissue covering visceral tissues that may compromise the physiological function of the whole digestive tract. This study reports a case of EPS caused by a complication of PD due to focal segmental glomerulosclerosis. In the case reported, the patient was submitted to PD and underwent two renal transplantes from living donor. Different surgical techniques were assessed, which have proved useful for clinical improvement of the patient. Parenteral nutrition has significantly contributed to the maintenance of nutrition, assisting in healing and in serum protein, vitamins and electrolytes. Tamoxifen therapy and administration of sodium hyposulfite were effective in retarding the progression of EPS.

Keywords: Peritonitis; Peritoneal dialysis; Bowel obstruction; Organ transplantation; Kidney transplantation; Peritoneal Fibrosis.

\section{REFERÊNCIAS}

1. Stuart S, Booth TC, Cash CJC, et al. Complications of continuous ambulatory peritoneal dialysis. Radiographics. 2009;29:441-60.

2. Rigby RJ, Hawley CM. Sclerosing peritonitis: the experience in Australia. Nephrol Dial Transplant 1998;13:154-9.

3. Kawaguchi $Y$, Kawanishi H, Mujais S, et al. Encapsulating peritoneal sclerosis: Definition, etiology, diagnosis, and treatment. Perit Dial Int. 2000;20:43-55.

4. Choi JH, Kim JH, Kim JJ, et al. Large bowel obstruction caused by sclerosing peritonitis: contrast-enhanced CT findings. Br J Radiol. 2004;77:344-6.

5. Bargman J, Harel Z. Encapsulating peritoneal sclerosis. US nephrol. 2011;5:71-5.

6. Habib AM, Preston E, Davenport A. Risk factors for developing encapsulating peritoneal sclerosis in the icodextrin era of peritoneal dialysis prescription. Nephrol Dial Transplant. 2010;25:1633-8.
7. Dabak R, Uygur-Bayramiçli O, Aydin DK et al. Encapsulating peritonitis and familial Mediterranean fever. Word J Gastroenteral. 2005. 11(18):2844-6.

8. Korte MR, Yo M, Betjes MG, et al. Increasing incidence of severe encapsulating peritoneal sclerosis after kidney transplantation. Nephrol Dial Transplant 2007;22:2412-4.

9. Betjes MGH SD, Lingsma $H$, Fieren $M$, et al. Risk factors associated with increased incidence of encapsulating peritoneal sclerosis in a controlled multicenter study. J Am Soc Nephrol. 2009; Abstract issue: 553776.

10. Nitsch D, Davenport A. Designing Epidemiology Studies to Determine the Incidence and Prevalence of Encapsulating Peritoneal Sclerosis (EPS). Perit Dial Int. 2015 Dec;35(7):678-82.

11. Kawanishi $H$, Harada $Y$, Noriyuki $T$, et al. Treatment options for encapsulating peritoneal sclerosis based on progressive stage. Advances in peritoneal dialysis. 2001;17:200-4. 
Vanessa Suemi Takenaka, Felipe Sbrolini Borges, Thales Franco de Andrade, André Petraroia Capelozza, Sibele Braga, Gabriel Castilho Schnorr, Laís Pacca Nicolellis, Jorge Marcelo Padilla Mancero, Irene L. Noronha, André Ibrahim David

12. Kawanishi $\mathrm{H}$, Moriishi $\mathrm{M}$, Ide $\mathrm{K}$, et al. Recommendation of the surgical option for treatment of encapsulating peritoneal sclerosis. Dial Int. 2008 Jun;28 (Suppl 3):S205-10.

13. Nomoto $Y$, Kawaguchi $Y$, Kubo H, et al. Sclerosing encapsulating peritonitis in patients undergoing continuous ambulatory peritoneal dialysis: a report of the Japanese Sclerosing Encapsulating Peritonitis Study Group. Am J Kidney Dis. 1996;28:420-7.

14. Ce'licout $B$, Levard $H$, Hay J et al. Sclerosing encapsulating peritonitis: Early and late results of surgical management in 32 cases. Dig Surgery. 1998;15(6):697-702.

15. Kawanishi H. Encapsulating peritoneal sclerosis (review). Nephrology 2005;10:249-5.

16. Kawanishi H, Moriishi M, Tsuchiya S. Experience of 100 surgical cases of encapsulating peritoneal sclerosis: Investigation of recurrent cases after surgery. Adv Perit Dial 2006;22:60-4

17. Margetts PJ, Bonniaud P, Liu L, et al. Transient overexpression of TGF- $\beta 1$ induces epithelial mesenchymal transition in the rodent peritoneum. J Am Soc Nephrol 2005;16:425-36.

18. Khanna A, Plummer M, Bromberek C, et al. Expression of TGFbeta and fibrogenic genes in transplant recipients with tacrolimus and cyclosporine nephrotoxicity. Kidney Int 2002;62:2257-63.

19. Nakamoto H. Encapsulating peritoneal sclerosis - a clinician's approach to diagnosis and medical treatment. Perit Dial Int. 2005;25(Suppl 4):S30-8.

20. Merkle M, Wörnle M. Sclerosing peritonitis: a rare but fatal complication of peritoneal inflammation. Mediators Inflamm 2012;2012:709673.

21. Wong CF, Beshir S, Khalil A, et al. Successful treatment of encapsulating peritoneal sclerosis with azathioprine and prednisolone.Perit Dial Int. 2005;25(3):285-7.

22. Bhandari S. Recovery of gastrointestinal function after renal transplantation in patients with sclerosing peritonitis secondary to continuous ambulatory peritoneal dialysis. Am J Kidney Dis. 1996;27(4):604.

23. Clark CP, Vanderpool D, Preskitt JT. The response of retroperitoneal fibrosis to tamoxifen. Surgery. 1991;109:502-6.

24. Loffeld RJ, Van Weel TF. Tamoxifen for retroperitoneal fibrosis. Lancet. 1993;341: 82
25. Van Bommel EF, Hendriksz TR, Huiskes AW, et al. Tamoxifen therapy for nonmalignant retroperitoneal fibrosis. Ann Intern Med. 2006;2:101-6.

26. Özener Ç, Kiris S, Lawrence R, et al. Potential beneficial effect of tamoxifen in retroperitoneal fibrosis. Nephrol Dial Transplant. 1997;12(10):2166-8.

27. Allaria PM, Giangrande A, Gandini E, et al. Continuous ambulatory peritoneal dialysis and sclerosing encapsulating peritonitis: tamoxifen as a new therapeutic agent? Nephrol. 1999;6:395-7.

28. Guest S. Tamoxifen therapy for encapsulating peritoneal sclerosis: mechanism of action and update on clinical experiences. Perit Dial Int. 2009;29(3):252-5

29. Korte MR, Fieren MW, Sampimon DE, et al. Tamoxifen is associated with lower mortality of encapsulating peritoneal sclerosis: results of the Dutch Multicentre EPS Study. Nephrol Dial Transplant. 2011;26:691-69

30. Wong CF. Clinical experience with tamoxifen in encapsulating peritoneal sclerosis. Perit Dial Int. 2006;26(2):183-4

31. Dellê H, Rocha JR, Cavaglieri RC, et al. Antifibrotic effect of tamoxifen in a model of progressive renal disease. J Am Soc Nephrol. 2012 Jan;23(1):37-48

32. Farah M, Crawford RI, Levin A, et al. Calciphylaxis in the current era: emerging 'ironic' features?. Nephrol Dial Transplant. 2011;26(1):191-5

33. Pinho A, Pinto I, Sampaio S, et al. Discovering implicit associations in a case of encapsulating peritoneal sclerosis complicated by severe mineral imbalance. BMJ Case Rep. 2014; Nov 3.

34. Cicone JS; Petronis JB; Embert CD; et al. Successful treatment of calciphylaxis with intravenous sodium thiosulfate. Am J Kidney Dis. 2004;43(6):1104-8.

35. Mataic D, Bastani B. Intraperitoneal sodium thiosulfate for the treatment of calciphylaxis. Ren Fail. 2006;28(4):361-3

36. Schlieper G, Brandenburg V, Ketteler M, et al. Sodium thiosulfate in the treatment of calcific uremic arteriolopathy. Nat Rev Nephrol. 2009 Sep;5(9):539-43. 